\title{
ON A CLASSIFICATION OF PLANE DOMAINS FOR BMOA
}

\author{
Dedicated to Professor Mitsuru Ozawa on the occasion of his 60th birthday
}

\section{BY SHŌJI KOBAYASHI}

1. Introduction. The space $B M O A$ is one which lies between the space $A B$ of bounded analytic functions and the Hardy class $H_{p}$ for any $p>0$. In this paper we are concerned with $B M O A$ for general domains and investigate the inclusion relations among the null classes $O_{A B}, O_{B M O A}$ and $O_{p}$ of plane domains corresponding to these spaces.

The space $B M O$ of functions of bounded mean oscillation was first introduced by John and Nirenberg [7], in the context of functions defied in $\boldsymbol{R}^{n}$. Since then several people $[1,3,5]$ investigated the space in various contexts and noticed that $B M O$ has deep connections with conjugate harmonic functions and the dual of Hardy class $H_{1}$. We state the definition of $B M O$ for functions defined on the unit circle $T$. Let $u$ be an integrable function on $T$ and $I$ be a subarc of $T$. We denote by $u_{1}$ the average of $u$ over $I$, that is,

$$
u_{I}=\frac{1}{|I|} \int_{I} u\left(e^{i t}\right) d t
$$

where $|I|$ denotes the Lebesgue measure of $I$. We say that $u$ is of bounded mean oscillation, $u \in B M O$, if

$$
\sup _{I} \frac{1}{|I|} \int_{I}\left|u\left(e^{\imath t}\right)-u_{I}\right| d t<+\infty,
$$

where the supremum is taken over all subarcs $I \subset T$. We denote by $B M O A$ the set of functions in $B M O$ whose Poisson extensions to the unit disc $D$ are analytic. It is known that $B M O A$ can be defined in an equivalent way which makes it conformally invariant.

Let $f$ be an analytic function in $D$. We use the following notations :

$$
\|f\|_{p}=\sup _{0<r<1}\left(\frac{1}{2 \pi} \int_{0}^{2 \pi}\left|f\left(r e^{i \theta}\right)\right|^{p} d \theta\right)^{1 / p}, \quad 0<p<\infty,
$$

and

$$
H_{p}(D)=\left\{f: f \text { is analytic in } D \text { and }\|f\|_{p}<+\infty\right\} \text {, }
$$

$$
T(f)=\sup _{0<r<1} \frac{1}{2 \pi} \int_{0}^{2 \pi} \log ^{+}\left|f\left(r e^{i \theta}\right)\right| d \theta .
$$

Received March 31, 1983 
It is known that for $f$ analytic in $D$ the following are equivalent (see, for example, [1] or [4]):

(a) $f \in B M O A$;

(b) $\sup _{a \in D} \iint_{D}\left|f^{\prime}(z)\right|^{2} \log \left|\frac{1-\bar{a} z}{z-a}\right| d x d y<+\infty$;

(c) $f(z)=f_{1}(z)+i f_{2}(z), z \in D$, for some $f_{j}$ analytic in $D$ with $\operatorname{Re} f_{j} \in H B(D)$ for $j=1,2$, where $H B$ denotes the space of bounded harmonic functions ;

(d) $\sup _{a \in D}\left\|f\left(\frac{z+a}{1+\bar{a} z}\right)-f(a)\right\|_{p}<+\infty$, for every $p>0$;

(e) $\sup _{a \in D}\left\|f\left(\frac{z+a}{1+\bar{a} z}\right)-f(a)\right\|_{p}<+\infty$, for some $p>0$;

(f) $\sup _{a \in D} T\left(f\left(\frac{z+a}{1+\bar{a} z}\right)-f(a)\right)<+\infty$.

In Section 2 we define $B M O A$ for general domains and state several equivalent conditions. In Section 3 we deal with a classification problem of plane domains for $B M O A$.

2. BMOA for general domains. Following Metzger [10], we defie $B M O A$ for general domains by using a similar condition to $(b)$. Let $G \notin O_{G}$ (i.e. $G$ possesses Green's function) be a domain in the extended complex plane $S$. We denote by $B M O A(G)$ the space of functions $f$ analytic in $G$ for which

$$
\sup _{a \in G} \iint_{G}\left|f^{\prime}(z)\right|^{2} g(z, a) d x d y<+\infty,
$$

where $g(z, a)$ denotes the Green's function of $G$ with pole at $a$.

Note that the condition (c) is not equivalent to (2.1) in the case where $G$ is not simply connected. As for conditions (d), (e) and (f), however, we can consider similar conditions for a general domain $G$, which are equivalent to (2.1). Let $S(G)$ denote the class of functions subharmonic in $G$, and following [9], for $u \in S(G)$ we denote by $\hat{u}$ the least harmonic majorant of $u$ in $G$, where we set $\hat{u}(z)=+\infty$ if $u$ admits no harmonic majorants.

THEOREM 1. For $f$ analytic in $G$, the following are equivalent:

(i) $f \in B M O A(G)$;

(ii) $\sup _{a \in G} \hat{u}_{a}(a)<+\infty$, where $u_{a}(z)=|f(z)-f(a)|^{p}$, for any $p>0$;

(iii) $\sup _{a \in G} \hat{u}_{a}(a)<+\infty$, where $u_{a}(z)=|f(z)-f(a)|^{p}$, for some $p>0$;

(iv) $\sup _{a \in G} \hat{u}_{a}(a)<+\infty$, where $u_{a}(z)=\log ^{+}|f(z)-f(a)|$. 
In order to prove the theorem, we need two lemmas, one of which is proved by using Green's theorem, and the other was essentially proved by Rudin [11, p. 48$]$.

LEMMA 1. For $f$ analytic in $G$ and $a \in G$ let $u_{a}(z)=|f(z)-f(a)|^{2}$, then

$$
\iint_{G}\left|f^{\prime}(z)\right|^{2} g(z, a) d x d y=\frac{\pi}{2} \hat{u}_{a}(a) .
$$

Proof. Let $\Omega$ be a plane domain with smooth boundary and let $u$ and $v$ be $C^{2}$ functions on $\bar{\Omega}$. Then Green's theorem states that

$$
\iint_{\Omega}(v \Delta u-u \Delta v) d x d y=\int_{\partial \Omega}\left(v \frac{\partial u}{\partial n}-u \frac{\partial v}{\partial n}\right) d s,
$$

where $\Delta$ denotes the Laplacian, $\frac{\partial}{\partial n}$ is differentiation in the outward normal direction, and $d s$ is the arc length element on $\partial \Omega$.

Let $\left\{G_{n}\right\}$ be a regular exaustion of $G$ such that $a \in G_{n}$ for $n=1,2, \cdots$. We apply (2.3) with $u(z)=|f(z)-f(a)|^{2}$ and $v(z)=g_{n}(z, a)$ in the domain obtained by delating from $G_{n}$ a small disc centered at $a$, where $g_{n}(z, a)$ denotes the Green's function of $G_{n}$. Noting $\Delta u=4\left|f^{\prime}(z)\right|^{2}$, we see by a simple calculation

$$
\begin{aligned}
\frac{2}{\pi} \iint_{G}\left|f^{\prime}(z)\right|^{2} g(z, a) d x d y & =\lim _{n \rightarrow \infty} \frac{2}{\pi} \iint_{G_{n}}\left|f^{\prime}(z)\right|^{2} g_{n}(z, a) d x d y \\
& =\lim _{n \rightarrow \infty} \frac{1}{2 \pi} \int_{\partial G_{n}}|f(z)-f(a)|^{2} \frac{\partial g_{n}(z, a)}{\partial n_{z}} d s \\
& =\hat{u}_{a}(a) .
\end{aligned}
$$

LEMma 2. Let $\pi$ be a universal covering map of $G$, then $\hat{f} \circ \pi=\widehat{f} \circ \pi$ for any $f \in S(G)$.

Proof. Since $\hat{f} \circ \pi$ is a harmonic majorant of $f \circ \pi$, we easily see that $\hat{f} \circ \pi$ $\geqq \widehat{f \circ \pi}$. We must show the inverse inequality. Let $\Gamma$ be the cover transformation group under which $\pi$ is invariant. Since $\widehat{f \circ \pi \circ T}$ is a harmonic majorant of $f \circ \pi \circ T=f \circ \pi$ for every $T \in \Gamma$, we see $\widehat{f \circ \pi} \widehat{f \circ \pi} \circ T$. By composing $T^{-1}$ from

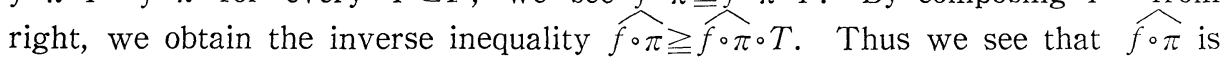
invariant under $\Gamma$. Therefore we can define a single-valued harmonic function in $G$ by $\widehat{f \circ \pi} \circ \pi^{-1}$, which is a harmonic majorant of $f$. Then we see that $\widehat{f \circ \pi} \pi^{-1}$ $\geqq \hat{f}$, and hence $\widehat{f} \circ \pi \geqq \hat{f} \circ \pi$, as asserted.

Proof of Theorem 1. By Lemma 1 we see that (2.1) and (iii) with $p=2$ are equivalent. In paticular, we see that (i) implies (iii) and that (ii) implies (i). It is obvious that (ii) implies (iii) and that (iii) implies (iv), since $\log ^{+} t \leqq t^{p} / p$ for any $t>0$ and $p>0$. 
All we must prove is that (iv) implies (ii). Suppose that (iv) holds and let $p$ be fixed with $0<p<\infty$. Let $g=f \circ \pi$ and $\varphi_{b}(z)=(z+b) /(1+\bar{b} z)$ for $b \in D$. Let $K_{1}=\sup _{a \in G} \hat{u}_{a}(a)$, where $u_{a}(z)=\log ^{+}|f(z)-f(a)|$. For every $b \in D$, set $v_{b}(z)$ $=\log ^{+}|g(z)-g(b)|$, and $a=\pi(b)$, then we see by Lemma 2

$$
\begin{aligned}
T\left(g\left(\frac{z+b}{1+\bar{b} z}\right)-g(b)\right) & \left.=\widehat{\left(v_{b}^{\circ} \varphi_{b}\right.}\right)(0) \\
& =\left(\hat{v}_{b}^{\circ} \varphi_{b}\right)(0) \\
& =\hat{v}_{b}(b) \\
& =\left(\widehat{u_{a} \circ \pi}\right)(b) \\
& =\left(\hat{u}_{a} \circ \pi\right)(b) \\
& =\hat{u}_{a}(a) \leqq K_{1},
\end{aligned}
$$

since $u_{a} \circ \pi=v_{b}$. Therefore we see that $g$ satisfies the condition (f), and hence (d), since these conditions are equivalent for functions analytic in $D$ as mentioned in the introduction.

Let $K_{2}=\sup _{b \in D}\|g((z+b) /(1+\bar{b} z))-g(b)\|_{p}$. For fixed $a \in G$, let $u_{a}(z)=|f(z)-f(a)|^{p}$ and take a point $b \in D$ such that $a=\pi(b)$, then we see again by Lemma 2

$$
\begin{aligned}
\hat{u}_{a}(a) & =\left(\hat{u}_{a} \circ \pi\right)(b) \\
& =\left(\widehat{u_{a} \circ \pi}\right)(b) \\
& =\hat{v}_{b}(b) \\
& =\left(\hat{v}_{b} \circ \varphi_{b}\right)(0) \\
& =\left(\widehat{v}_{b}^{\circ} \varphi_{b}\right)(0) \\
& =\left\|g\left(\frac{z+b}{1+\bar{b} z}\right)-g(b)\right\|_{p}^{p} \leqq K_{2}^{p},
\end{aligned}
$$

where $v_{b}(z)=|g(z)-g(b)|^{p}$. Therefore $f$ satisfies (ii), as asserted.

From the proof of the theorem we obtain

Corollary. For $f$ analytic in $G, f \in B M O A(G)$ if and only if $f \circ \pi \in B M O A(D)$.

Remark. Metzger [10] essentially proved the corollary in the way to showing $A D(G) \subset B M O A(G)$, by using Myrberg's theorem, but (the author thinks that) our proof is rather elementary. Here $A D(G)$ denotes the space of analytic functions with finite Dirichlet integrals in $G$. 
3. Classification of domains. Let $A B(G)$ denote the space of all bounded analytic functions in $G$, and $H_{p}(G), 0<p<\infty$, the Hardy class, denote the space of analytic functions $f$ for which $|f|^{p}$ admits a harmonic majorant in $G$. Note that when $G=D$ this definition is equivalent with (1.1). We denote by $O_{A B}$ (resp. $O_{B \text { MOA }}, O_{p}$ ) the set of all plane domains $G$ for which $A B(G)$ (resp. $\left.B M O A(G), H_{p}(G)\right)$ contains only the constants. By Theorem 1 we easily see that

$$
A B(G) \subset B M O A(G) \subset H_{p}(G),
$$

for any $G$ and any $p>0$, and hence

$$
O_{A B} \supset O_{B . Y O A} \supset \bigcup_{0<p<\infty} O_{p} .
$$

In this section we deal with a classification problem which asks whether the inclusion relation in (3.1) are strict or not. We denote by the sign of inequality $>$ a strict inclusion relation, and by $\operatorname{Cap}(E)$ the logarithmic capacity of a compact set $E$.

\section{THEOREM 2. $O_{A B}>O_{B M O A}>\bigcup_{0<p<\infty} O_{p}$.}

Proof. In order to prove $O_{A B}>O_{B . M O A}$, we must construct a plane domain $G$ for which $A B(G)$ contains only the constants while $B . M O A(G)$ contains a nonconstant function. Let $A$ be a compact totally disconnected set with $0 \notin A$ which lies on the interval $[-1,1] \operatorname{such}$ that $\operatorname{Cap}(A)>0$ but of linear measure 0 . For example, we can take as $A$ a Cantor ternary set which is constructed on the interval $[-1,1]$. Let $E_{n, m}=\{z+4 n+4 m i: z \in A\}$ for every integer $n$ and $m$, and $E=\bigcup_{n, m=-\infty}^{\infty} E_{n, m}$. Finally let $G$ be the complement of $E, G=C-E$, then $G$ is a plane domain with $0 \in G$.

We easily see that $E$ is removable for $A B$ functions, since it is a countable union of sets of linear measure 0 , and hence $G \in O_{A B}$. To show $G \notin O_{B M O A}$, we prove that $f(z)=z$ belongs to $B M O A(G)$. This follows from a deep result on $B M O A$ and omitted values by Hayman and Pommerenke [5], but we give another proof so as to make this paper self-contained. Let $F=\cup_{n=-\infty}^{\infty} E_{n}$, where $E_{n}=\{z+4 n: z \in A\}$, and $G_{1}=C-F$. The author [8] used the following lemma for $H_{p}$ classification.

Lemma 3. For every $p$ with $0<p<1, f(z)=z$ belongs to $H_{p}\left(G_{1}\right)$.

Proof. By a theorem of Kolmogorov [2, p. 57], every analytic function $g$ for which $|\operatorname{Im} g|$ admits a harmonic majorant belongs to $H_{p}$ for $0<p<1$. Therefore it is sufficient to prove that $|\operatorname{Im} z|$ admits a harmonic majorant in $G_{1}$. Let $\chi$ be the bounded harmonic function in $G_{1} \cap\{z: \operatorname{Im} z<2\}$ with boundary value 0 on $F$ and 1 on the line $\{z: \operatorname{Im} z=2\}$. Since $\operatorname{Cap}(F)>0$, we see that $\chi$ is nonconstant. Since $F$ is invariant under the translation $\phi(z)=z-4$, so is $\chi$. Therefore we see that 


$$
\sup \{\chi(z): \operatorname{Im} z=1\}=\max \{\chi(z): \operatorname{Im} z=1,-2 \leqq \operatorname{Re} z \leqq 2\} \leqq 1-\varepsilon
$$

for some $\varepsilon$ with $0<\varepsilon<1 / 2$, and hence we see

$$
\varepsilon^{-1} \chi(z)+2 \leqq \operatorname{Im} z+\varepsilon^{-1},
$$

on the line $\{z: \operatorname{Im} z=1\}$. Since (3.2) holds in equality on the line $\{z: \operatorname{Im} z=2\}$, we see that (3.2) holds in $\{z: 1<\operatorname{Im} z<2\}$ by the maximum principle. We define a positive function $s$ in $G_{1}$ by

$$
s(z)=\left\{\begin{array}{lll}
\operatorname{Im} z+\varepsilon^{-1} & \text { if } & \operatorname{Im} z \geqq 2, \\
\varepsilon^{-1} \chi(z)+2 & \text { if } & \operatorname{Im} z<2 .
\end{array}\right.
$$

If we can prove that $s$ is superharmonic in $G_{1}$, we see that $s(z)+s(\bar{z})$ is a superharmonic majorant of $|\operatorname{Im} z|$. Since $|\operatorname{Im} z|$ is subharmonic, we easily see that $|\operatorname{Im} z|$ admits a harmonic majorant in $G_{1}$ by the Perron's family argument. Therefore it is sufficient to prove that $s$ is superharmonic on the line $\{z: \operatorname{Im} z=2\}$, since $s$ is harmonic off the line. Fix any $z_{0}$ with $\operatorname{Im} z_{0}=2$ and $r$ with $0<r<1$, then we see by (3.2) and (3.3)

$$
\begin{aligned}
s\left(z_{0}\right) & =\operatorname{Im} z_{0}+\varepsilon^{-1} \\
& =\frac{1}{2 \pi} \int_{0}^{2 \pi}\left(\operatorname{Im}\left(z_{0}+r e^{i \theta}\right)+\varepsilon^{-1}\right) d \theta \\
& \geqq \frac{1}{2 \pi}\left(\int_{0}^{\pi}\left(\operatorname{Im}\left(z_{0}+r e^{i \theta}\right)+\varepsilon^{-1}\right) d \theta+\int_{\pi}^{2 \pi}\left(\varepsilon^{-1} \chi\left(z_{0}+r e^{i \theta}\right)+2\right) d \theta\right) \\
& =\frac{1}{2 \pi} \int_{0}^{2 \pi} s\left(z_{0}+r e^{i \theta}\right) d \theta,
\end{aligned}
$$

and hence $s$ is superharmonic at $z_{0}$, as asserted. This completes the proof of the lemma.

Let $p$ be arbitrarily fixed with $0<p<1$, and put $u_{a}(z)=|z-a|^{p}$ for $a \in G$. By Lemma 3 we see that $u_{a}$ admits a harmonic majorant in $G$, since $G \subset G_{1}$. Let $\omega=4 n+4 m i$, where $m$ and $n$ are integers, then we see that

$$
u_{a+\omega}(z)=u_{a}(z-\omega) \leqq \hat{u}_{a}(z-\omega)
$$

for $z \in G$, since $G$ is invariant under the translation $\phi(z)=z+\omega$. Therefore we obtain

and hence

$$
\hat{\imath}_{a+\omega}(z) \leqq \hat{u}_{a}(z-\omega),
$$

$$
\hat{u}_{a+\omega}(a+\omega) \leqq \hat{u}_{a}(a) .
$$

By putting $a=a-\omega$ and then $\omega=-\omega$ in (3.4), we obtain the inverse inequality, and hence

$$
\widehat{u}_{a+\omega}(a+\omega)=\hat{u}_{a}(a),
$$


which means that $\hat{u}_{a}(a)$ is invariant under the translation $\phi(a)=a+\omega$ as a function of $a$. Since $u_{a}(z) \leqq 2^{p}\left(|z|^{p}+|a|^{p}\right)=2^{p}\left(u_{0}(z)+|a|^{p}\right)$, we see

$$
\hat{u}_{a}(a) \leqq 2^{p}\left(\hat{u}_{0}(a)+|a|^{p}\right)
$$

for every $a \in G$. Let $Q=\{z:|\operatorname{Re} z|<2,|\operatorname{Im} z|<2\}$ and $M=\max _{z \in \partial Q} \hat{u}_{0}(z)$. If we define

$$
v(z)= \begin{cases}\min \left(\hat{u}_{0}(z), M\right) & \text { for } z \in Q \cap G, \\ \hat{u}_{0}(z) & \text { for } z \in Q^{c} \cap G,\end{cases}
$$

then we easily see that $v(z)$ is a superharmonic majorant of $u_{0}(z)$, since $u_{0}(z) \leqq M$ for $z \in Q$ by the maximum principle. Therefore we see that

$$
\hat{\imath}_{0}(z) \leqq v(z) \leqq M
$$

for $z \in Q \cap G$. By (3.5), (3.6) and (3.7) we obtain

$$
\begin{aligned}
\sup _{a \in G} \hat{u}_{a}(a) & =\sup _{a \in Q \cap G} \hat{u}_{a}(a) \\
& \leqq 2^{p} \sup _{a \in Q \cap G}\left(\hat{u}_{0}(a)+|a|^{p}\right) \\
& \leqq 2^{p}\left(M+8^{1 / 2 p}\right)<+\infty,
\end{aligned}
$$

which means that $f(z)=z$ satisfies the condition (iii) of Theorem 1, and hence $f(z)=z$ belongs to $B M O A(G)$ by Theorem 1 , as asserted.

Next we prove $O_{B M O A}>\bigcup_{0<p<\infty} O_{p}$. For this we must construct a domain $G$ for which $B M O A(G)$ contains only the constants while $H_{p}(G)$ contains a nonconstant function for every $p$ with $0<p<\infty$.

It is known that $O_{p}>O_{q}$ if $p>q \geqq 1$ ([8]). Let $E_{k}$ be a compact totally disconnected set which satisfies

$$
S-E_{k} \in O_{k+1}-O_{k}
$$

Since the condition (3.8) remains unchanged if $E_{k}$ is mapped by a parallel translation or a homothetic transformation, we can take $E_{k}$ so that $E_{k}$ is contained in the disc $\left\{z:\left|z-4^{k}\right| \leqq 1\right\}$ for $k=1,2, \cdots$. Let $E=\bigcup_{k=1}^{\infty} E_{k}$ and $G$ be the complement of $E, G=C-E$. It is trivial that $G \notin O_{p}$ for every $p>0$, since $G \subset S-E_{k}$ for $k=1,2, \cdots$.

In order to prove $G \in O_{B M O A}$, we suppose $f \in B M O A(G)$ and show that $f$ is constant. Since $B M O A(G) \subset H_{p}(G), f$ belongs to $H_{p}(G)$ for every $p>0$. Therefore we see that every point on $E_{k}$ is removable singularity of $f$, and hence $f$ is analytic in the whole complex plane $\boldsymbol{C}$. Then $f$ can be expressed as a Taylor expansion

$$
f(z)=\sum_{m=0}^{\infty} c_{m} z^{m}
$$


for $z \in \boldsymbol{C}$. Let

$$
G_{k}=\left\{z:|z|<3 \cdot 4^{k}\right\}-\left\{z:\left|z-4^{k}\right| \leqq 4^{k}\right\}
$$

and $g_{k}(z)=g_{k}\left(z,-4^{k}\right)$ be the Green's function of $G_{k}$ with pole at $-4^{k}$ for $k=0,1,2, \cdots$. Since $G_{k}=4^{k} G_{0} \equiv\left\{4^{k} z ; z \in G_{0}\right\}$ by (3.10), we see

and

$$
g_{k}(z)=g_{0}\left(4^{-k} z\right)
$$

$$
\frac{\partial g_{k}(z)}{\partial n}=4^{-k} \frac{\partial g_{0}\left(4^{-k} z\right)}{\partial n}
$$

for $z \in \partial G_{k}$, where $\frac{\partial}{\partial n}$ denotes differentiation in the inward normal direction. Let $u_{a}(z)=|f(z)-f(a)|$ for $a \subseteq G$ and write $a_{k}=-4^{k}$. If we put

$$
\varepsilon=\min _{z \in \partial G_{0}} \frac{\partial g_{0}(z)}{\partial n}>0
$$

then we see by (3.11) that

$$
\frac{\partial g_{k}(z)}{\partial n} \geqq 4^{-k} \varepsilon
$$

on $\partial G_{k}$. Therefore we see

$$
\begin{aligned}
\hat{\varkappa}_{a_{k}}\left(a_{k}\right) & \geqq \frac{1}{2 \pi} \int_{\partial G_{k}}\left|f(z)-f\left(a_{k}\right)\right| \frac{\partial g_{k}(z)}{\partial n}|d z| \\
& \geqq \frac{4^{-k} \varepsilon}{2 \pi} \int_{|z|=3 \cdot 4 k}\left|f(z)-f\left(a_{k}\right)\right||d z|,
\end{aligned}
$$

since $G_{k} \subset G$ for $k=0,1,2, \cdots$. Since $f$ satisfies the condition (iii) of Theorem 1 with $p=1$, we see that

$$
\frac{1}{2 \pi} \int_{|z|=3 \cdot 1 k}\left|f(z)-f\left(a_{k}\right)\right||d z| \leqq 4^{k} C
$$

for some constant $C$. It is well known that the coefficient $c_{m}$ in the expansion (3.9) is expressed as

$$
\begin{aligned}
c_{m} & =\frac{1}{2 \pi i} \int_{|z|=R} \frac{f(z)}{z^{m+1}} d z \\
& =\frac{1}{2 \pi i} \int_{|z|=R} \frac{f(z)-f\left(a_{k}\right)}{z^{m+1}} d z
\end{aligned}
$$

for $m=1,2, \cdots$, where $R$ is an arbitrary positive number. Putting $R=3 \cdot 4^{k}$ in (3.13) and using (3.12), we obtain

$$
\begin{aligned}
\left|c_{m}\right| & \leqq \frac{1}{2 \pi} \int_{|z|=3 \cdot 4^{k}} \frac{\left|f(z)-f\left(a_{k}\right)\right|}{\left(3 \cdot 4^{k}\right)^{m+1}}|d z| \\
& \leqq C /\left(3^{m+1} \cdot 4^{k m}\right) .
\end{aligned}
$$


Letting $k \rightarrow \infty$, we see that $c_{m}=0$ for $m=1,2, \cdots$, and hence $f$ is constant, as asserted. This completes the proof of the theorem.

4. Concluding remarks. It is easily seen that the null class of plane domains corresponding to the space of analytic functions which satisfy the condition (c) coincides with $O_{A B}$. In fact, if $G \in O_{A B}$ and if a function $f$ analytic in $G$ is expressed as $f(z)=f_{1}(z)+i f_{2}(z), z \in G$, with $\operatorname{Re} f_{j} \in H B(G)$ for $j=1,2$, then $g_{j}(z)=\exp f_{j}(z)$ belongs to $A B(G)$. Therefore $g_{j}$ is constant and so is $f_{j}$ for $j=1,2$, and hence $f$ is also constant. (cf. [5, p. 220])

Let $E$ be the set which we used to prove $O_{A B}>O_{B M O A}$. Let $F=\log (E)$, the image of $E$ under all branches of $\log$, and $G=C-F$. Then we easily show that $G \in O_{A B}-O_{B M O A}$ and that $f(z)=e^{z}$ belongs to $B M O A(G)$. Therefore we can construct a plane domain $G \in O_{A B}-O_{B M O A}$ which does not satisfy the geometric condition of Hayman and Pommerenke's theorem [5] and for which $B M O A(G)$ contains a function with exponential growth.

Let $G$ be the domain which we used to prove $O_{B M O A}>\bigcup_{0<p<\infty} O_{p}$. By modifying somewhat our proof, we can also prove that if $f \in H_{1}(G)$ and $f$ is analytic in the whole plane $\boldsymbol{C}$, then $f$ is constant.

\section{REFERENCES}

[1] Baernstein, A., Analytic functions of bounded mean oscillation, Aspects of contemporary complex analysis, 1980, Academic Press, 3-36.

[2] Duren, P.L., Theory of $H^{p}$ spaces, 1970, Academic Press.

[3] Fefferman, C., Characterizations of bounded mean oscillation, Bull. Amer. Math. Soc. 77 (1971), 587-588.

[4] Garnett, J., Bounded analytic functions, 1981, Academic Press.

[5] Hayman, W.K. And Pommerenke, C., On analytic functions of bounded mean oscillation, Bull. London Math. Soc. 19 (1978), 219-224.

[6] Hejhal, D. A., Classification theory for Hardy classes of analytic functions, Ann. Acad. Sci. Fenn. A. I. 566 (1973), 1-28.

[7] John, F. And Nirenberg, L., On functions of bounded mean oscillation, Comm. Pure Appl. Math. 14 (1961), 415-426.

[8] Kobayashi, S., On a classification of plane domains for Hardy classes, Proc. Amer. Math. Soc. 68 (1978), 79-82.

[9] Kobayashi, S. ANd Suita, N., On subordination of subharmonic functions, Kodai Math. J. 3 (1980), 315-320.

[10] Metzger, T.A., On BMOA for Riemann surfaces, Can. J. Math. 18 (1981), 12551260.

[11] Rudin, W., Analytic functions of class $H_{p}$, Trans. Amer. Math. Soc. 78 (1955), 46-66.

Department of Electric Engineering

Technological University of Nagaoka 